\title{
Uso da ivermectina no tratamento da COVID-19 em humanos: revisão integrativa
}

\author{
Use of ivermectin in the treatment of COVID-19 in humans: integrative review \\ Uso de ivermectina en el tratamiento de COVID-19 en humanos: revisión integrativa
}

Recebido: 20/09/2021 | Revisado: 26/09/2021 | Aceito: 28/09/2021 | Publicado: 01/10/2021

\begin{abstract}
Nicoli Maciel
ORCID: https://orcid.org/0000-0003-1622-5135 Sociedade Educacional de Santa Catarina, Brasil E-mail: maciel.nicoli@gmail.com Vivian Binder Neis ORCID: https://orcid.org/0000-0001-6328-431X Sociedade Educacional de Santa Catarina, Brasil E-mail: vivian.neis@prof.unisociesc.com.br

Adrielli Tenfen

ORCID: https://orcid.org/0000-0003-3554-2152 Sociedade Educacional de Santa Catarina, Brasil E-mail: adrielli.voltolini@unisociesc.com.br
\end{abstract}

\begin{abstract}
Resumo
Com o aparecimento dos primeiros casos de infecção pelo SARS-CoV-2, a OMS declarou rapidamente pandemia frente a COVID-19, devido à alta velocidade e transmissibilidade da doença. Nesse cenário, além das pesquisas para o desenvolvimento de novos fármacos e vacinas, passou-se também a estudar medicamentos já existentes e aprovados, através do reposicionamento de fármacos, como alternativa para o tratamento da COVID-19, como é o caso da ivermectina. Desde então, vários ensaios clínicos estão sendo realizados ao redor do mundo para avaliar a ação antiviral da ivermectina frente a COVID-19. Dessa forma, essa revisão integrativa tem por objetivo analisar a literatura científica que trata de ensaios clínicos sobre o uso da ivermectina como tratamento para a COVID-19, descrevendo os trabalhos, bem como, discutindo seus resultados e limitações. Procedeu-se a busca de estudos científicos publicados nas bases eletrônicas de dados: PubMed, LILACS e SciELO, utilizando as palavras chaves "IVERMECTIN and COVID-19" e "IVERMECTIN and SARS-COV-2". Selecionou-se artigos publicados a partir de 2019, que trabalharam o emprego da ivermectina como tratamento para a COVID-19. A pesquisa encontrou estudos que demonstraram resultados tanto positivos quanto negativos para o uso da ivermectina frente alguns parâmetros do tratamento da COVID-19. Todavia, os trabalhos de forma geral, apresentam limitações metodológicas. Dessa forma, a evidência atual sobre o uso desse medicamento para tratar pacientes com COVID-19 é inconclusiva, indicando a necessidade de novos ensaios clínicos bem desenhados e estruturados, para confirmar ou descartar essas descobertas.
\end{abstract}

Palavras-chave: Ivermectina; COVID-19; Tratamento; Estudos clínicos.

\begin{abstract}
With the appearance of the first cases of infection by SARS-CoV-2, the WHO quickly declared a pandemic against COVID-19, due to the high speed and transmissibility of the disease. In this scenario, in addition to research for the development of new drugs and vaccines, existing and approved drugs have also been studied, through the repositioning of drugs, as an alternative for the treatment of COVID-19, as is the case with ivermectin. Since then, several clinical trials have been carried out around the world to evaluate the antiviral action of ivermectin against COVID-19. Thus, this integrative review aims to analyze the scientific literature dealing with clinical trials on the use of ivermectin as a treatment for COVID-19, describing the works, as well as discussing their results and limitations. The search for scientific studies published in electronic databases: PubMed, LILACS and SciELO was carried out, using the keywords "IVERMECTIN and COVID-19" and "IVERMECTIN and SARS-COV-2". Articles published from 2019 onwards, which worked on the use of ivermectin as a treatment for COVID-19, were selected. The research found studies that showed both positive and negative results for the use of ivermectin in relation to some parameters of the treatment of COVID-19. However, the works in general have methodological limitations. Thus, current evidence on the use of this drug to treat patients with COVID-19 is inconclusive, indicating the need for further welldesigned and structured clinical trials to confirm or rule out these findings.
\end{abstract}

Keywords: Ivermectin; COVID-19; Treatment; Clinical studies.

\section{Resumen}

Con la aparición de los primeros casos de infección por SARS-CoV-2, la OMS rápidamente declaró una pandemia contra COVID-19, debido a la alta velocidad y transmisibilidad de la enfermedad. En este escenario, además de la investigación para el desarrollo de nuevos medicamentos y vacunas, también se estudiaron medicamentos existentes y 
aprobados, a través del reposicionamiento de medicamentos, como alternativa para el tratamiento del COVID-19, como es el caso de la ivermectina. Desde entonces, se han realizado varios ensayos clínicos en todo el mundo para evaluar la acción antiviral de la ivermectina contra COVID-19. Así, esta revisión integradora tiene como objetivo analizar la literatura científica que trata sobre ensayos clínicos sobre el uso de ivermectina como tratamiento para el COVID-19, describiendo los trabajos, así como discutiendo sus resultados y limitaciones. Se realizó la búsqueda de estudios científicos publicados en bases de datos electrónicas: PubMed, LILACS y SciELO, utilizando las palabras clave "IVERMECTIN y COVID-19" e "IVERMECTIN y SARS-COV-2". Se seleccionaron los artículos publicados a partir de 2019 que trabajaban sobre el uso de ivermectina como tratamiento para COVID-19. La investigación encontró estudios que mostraron resultados tanto positivos como negativos para el uso de ivermectina en relación con algunos parámetros del tratamiento de COVID-19. Sin embargo, los trabajos en general tienen limitaciones metodológicas. Por lo tanto, la evidencia actual sobre el uso de este medicamento para tratar a pacientes con COVID19 no es concluyente, lo que indica la necesidad de más ensayos clínicos bien diseñados y estructurados para confirmar o descartar estos hallazgos.

Palabras clave: Ivermectina; COVID-19; Tratamiento; Estudios clínicos.

\section{Introdução}

Em dezembro de 2019, foram reportados casos de pneumonia aguda de etiologia desconhecida em Wuhan, na China. Contudo, após o isolamento e identificação do agente etiológico, a causa da doença foi atribuída a um novo $\beta$-coronavírus, que inicialmente foi chamado de novo coronavírus 2019 (2019-nCoV) pela Organização Mundial da Saúde (OMS) (Samaddar et al., 2020; Dos Santos, 2020).

Em fevereiro de 2020, a OMS nomeou oficialmente a doença como doença do coronavírus 2019 (COVID-19), ao mesmo passo que o Grupo de Estudos do Coronavírus, do Comitê Internacional de Taxonomia de Vírus, denominou o novo coronavírus como síndrome respiratória grave (SARS-CoV-2), pela semelhança com o coronavírus responsável pelo surto em 2003 (Guo et al., 2020; Dos Santos, 2020).

A OMS declarou pandemia pela COVID-19 em 11 março de 2020, devido a velocidade e escala de transmissão da doença (Dos Santos, 2020). Até 31 de março de 2020, já havia 750.890 casos confirmados e 36.405 mortes no mundo. Mais de um ano depois, em 31 de maio de 2021, haviam 169.597.415 casos confirmados, com 3.530.582 de mortes acumuladas no mundo (World Health Organization [WHO], 2021).

Dessa forma, surgiu a necessidade do desenvolvimento de medicamentos antivirais e vacinas para a COVID-19 (Trivedi et al., 2020). O advento das tecnologias genômicas e computacionais permitiram que em poucos meses já houvessem ensaios clínicos para vacinas e terapias medicamentosas em todo mundo. Além disso, se passou a estudar medicamentos já existentes e aprovados, através da estratégia de reposicionamento de fármacos, como alternativa para o tratamento da COVID19, como é o caso da ivermectina (Lima et al., 2020; Mishra \& Triphati, 2021; Portmann-Baracco et al., 2020).

A ivermectina foi descoberta em 1967 no Japão e foi introduzida na prática clínica em 1987 (Kaur et al., 2021). Ela é utilizada a mais de 30 anos para o tratamento de diferentes tipos de infecções parasitárias em humanos (Pandey et al., 2020), como filariose, estrongiloidíase, ascaridíase, sarna, pediculose e rosácea (Jans \& Wagstaff, 2020). Todavia, o espectro de atividade da ivermectina cresce todos os anos (Sharun et al., 2019). Hoje sabe-se que ela também possui ação na tripanossomíase, miíase orbitária, triquinose, leishmaniose, para o controle do inseto vetor da malária, também possui atividade antibacteriana e anticâncer (Kaur et al., 2021; Li et al., 2020).

Desde 2012, estudos demonstram que a ivermectina possui propriedades antivirais contra uma série de RNA virais, incluindo o vírus da dengue, imunodeficiência humana (HIV), Zika, febre amarela, chikunguya e mais recentemente começouse a discutir a ação antiviral da ivermectina frente ao SARS-CoV-2 (Heidary \& Gharebaghi, 2020; Jans \& Wagstaff, 2020).

A atividade antiviral da ivermectina está relacionada com a inibição da função de transporte nuclear da importina $\alpha / \beta 1$ (IMP $\alpha / \beta 1$ ), uma proteína responsável por reconhecer sequências de localização nuclear durante a infecção, no fechamento 
nucleocitoplasmático dependente de sinal da proteína do núcleocapsídeo do SARS-CoV, afetando a divisão celular da célula hospedeira (Hamed, 2020; Wulan et al., 2015).

Em março de 2020, Caly et al. (2020) realizaram um estudo in vitro para avaliar a atividade antiviral da ivermectina diante do SARS-CoV-2. O estudo demonstrou que após 48 horas da administração de $5 \mu \mathrm{M}$ de ivermectina em células Vero/hsLAM infectadas com SARS-CoV-2, houve uma redução de 5000 vezes do RNA viral em relação as amostras controle. Em contrapartida, a partir desse trabalho, outro estudo demonstrou que a concentração inibitória média ( $\left.\mathrm{IC}_{50} ; 2 \mu \mathrm{M}\right)$ era 35 vezes maior que a concentração plasmática máxima $\left(\mathrm{C}_{\max }\right)$ após a administração da dose aprovada de ivermectina (Schmith et al., 2020).

Desde então, vários ensaios clínicos estão sendo realizados ao redor do mundo para avaliar a ação antiviral da ivermectina frente a COVID-19. Dessa forma, o objetivo dessa revisão é analisar a literatura científica que trata de ensaios clínicos sobre o uso da ivermectina como tratamento alternativo para COVID-19, descrevendo os trabalhos, bem como, discutindo seus resultados e limitações.

\section{Metodologia}

Trata-se de uma revisão integrativa da literatura, metodologia que possibilita agrupar e sintetizar as pesquisas disponíveis sobre um tema, permitindo estabelecer conclusões gerais sobre a temática, direcionando a prática clínica e a tomada de decisões fundamentadas em evidências científicas. Para a elaboração dessa revisão foram consideradas seis etapas: identificação do tema e elaboração da pergunta norteadora, busca ou amostragem na literatura através de critérios de inclusão e exclusão definidos, coleta de dados, análise crítica dos estudos incluídos, discussão dos resultados e apresentação da revisão integrativa (Köche, 2011; Mendes et al., 2019; Souza et al., 2010).

A questão norteadora elaborada para guiar essa revisão integrativa foi: "O uso da ivermectina é eficaz para o tratamento da COVID-19?". Para a seleção da amostra, procedeu-se uma busca de estudos científicos publicados sobre a temática nas seguintes bases eletrônicas de dados: Biblioteca Nacional de Medicina (PubMed), Ciências Sociais e da Saúde da América Latina e do Caribe (LILACS) e Biblioteca Eletrônica Científica Online (SciELO). Para a pesquisa utilizou-se as palavras chaves "IVERMECTIN and COVID-19" e "IVERMECTIN and SARS-COV-2".

O período das buscas se deu entre março de 2021 e início de junho de 2021. Foram incluídos estudos clínicos originais publicados a partir de 2019, podendo ser produções em inglês, português ou espanhol e sem delimitação de país de publicação, que trabalharam o emprego da ivermectina como tratamento para a COVID-19. Em contrapartida, foram excluídos artigos que não atendessem ao objetivo do estudo, resumos expandidos, revisões bibliográficas e metanálises, estudos duplicados, além de trabalhos que tinham um enfoque maior em outros medicamentos ou combinação de vários tratamentos sem enfoque significativo na ivermectina, bem como, o uso profilático da ivermectina na COVID-19. O processo de seleção dos artigos, bem como os resultados, está apresentado na Figura 1.

A seleção dos trabalhos publicados se deu em duas etapas. Em um primeiro momento, o total de artigos encontrados pela estratégia de busca foram primeiramente filtrados através da avaliação do título e resumo, realizando-se uma pré-seleção daqueles que eram estudos clínicos. Em um segundo momento, procedeu-se a leitura e avaliação desses trabalhos na íntegra, para a confirmação da elegibilidade através dos critérios de inclusão e exclusão pré-estabelecidos. 
Figura 1. Fluxograma da busca e seleção dos artigos.

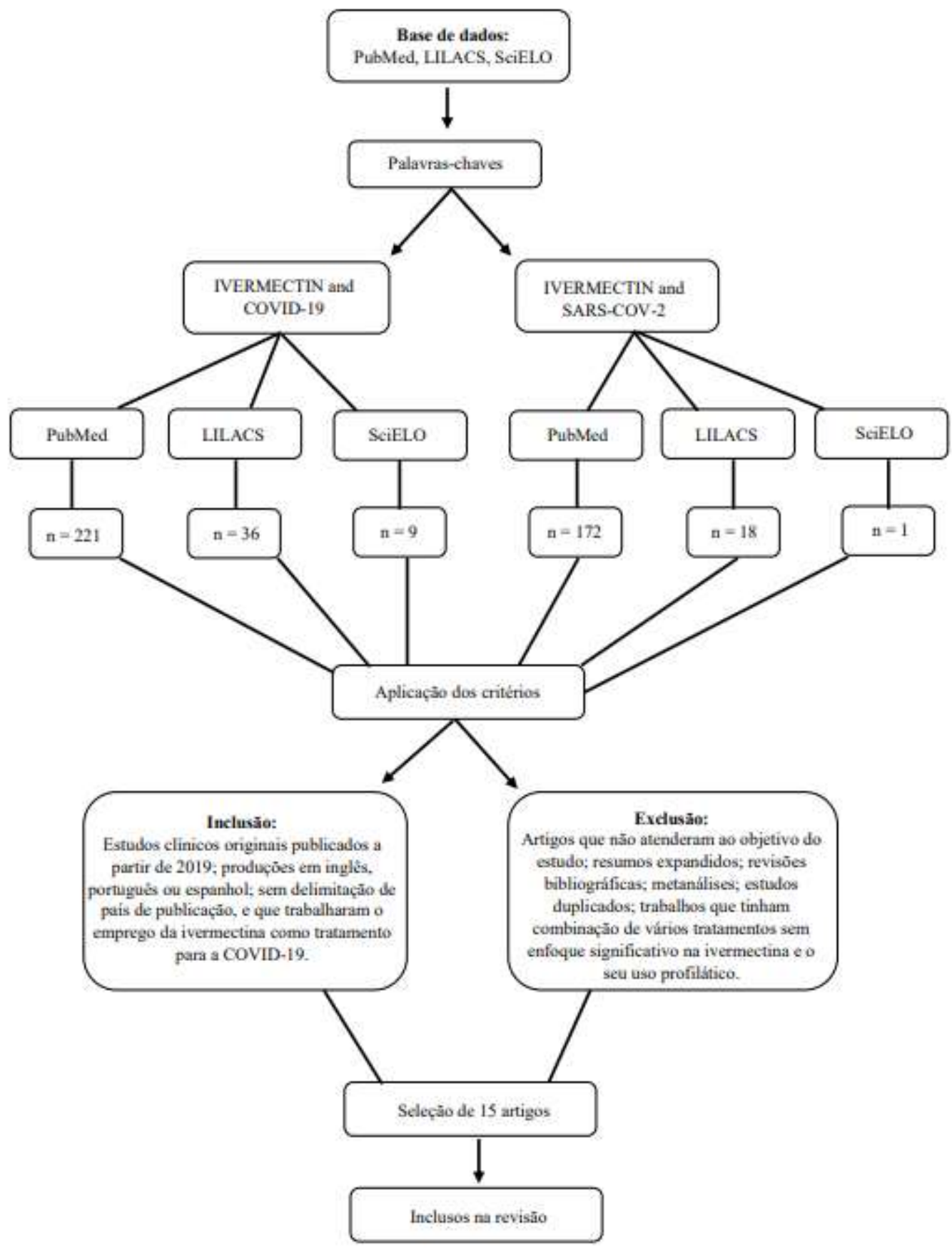

Fonte: Autores (2021).

\section{Resultados}

Através da utilização das palavras chaves "IVERMECTIN and COVID-19", foram encontrados 221 artigos na PubMed, 36 no LILACS e 9 na base de dados do SciELO. Em contrapartida, ao realizar a busca através de "IVERMECTIN and SARS-COV-2”, encontrou-se 172 estudos da PubMed, 18 no LILACS e 1 no SciELO. Após, através da aplicação dos critérios de inclusão e exclusão, selecionou-se 15 artigos que cumpriram os critérios estabelecidos, para integrar essa revisão. A amostra final, com título, autores, intervenção, tipo de estudo e local estão resumidos no Quadro 1, dividindo os nove 
estudos que evidenciam resultados positivos e os seis que mostram resultados negativos no uso da ivermectina para o tratamento da COVID-19.

Quadro 1. Estudos clínicos sobre o uso da ivermectina no tratamento da COVID-19 no período de 2019 a 2021.

\begin{tabular}{cccc}
\hline TÍTULO & AUTORES & INTERVENÇÃO & TIPO DE \\
ESTUDO & LOCAL \\
\hline & ESTUDOS COM RESULTADOS POSITIVOS PARA IVERMECTINA VS. COVID-19 \\
\hline
\end{tabular}

O tratamento com ivermectina pode melhorar o prognóstico de pacientes com COVID-19

\section{Khan et al.} (2020)

\author{
Ivermectina $12 \mathrm{mg}$ dentro de 24 horas após a \\ admissão hospitalar mais TP vs. TP.
}

\author{
Estudo \\ retrospectivo
}

Bangladesh

\begin{abstract}
O uso da Ivermectina está associado a uma baixa mortalidade em pacientes hospitalizados com a Doença do Coronavírus 2019. A

Ivermectina na COVID estudo dezenove
\end{abstract}

Uma dose de ivermectina $200 \mathrm{mcg} / \mathrm{kg}$ (sendo passível uma segunda dose no $7^{\circ}$ dia de
Rajter et al. (2021) tratamento) mais tratamento usual vs. tratamento usual (sem ivermectina). A maioria dos pacientes em ambos os grupos também recebeu corticosteroides, hidroxicloroquina e/ou azitromicina.

$\begin{array}{cc}\text { Estudo de } & \text { Flórida }- \\ \text { coorte } & \text { Estados } \\ \text { retrospectivo } & \text { Unidos }\end{array}$

Uso de Ivermectina no tratamento da Covid-19: um ensaio piloto
Pott-Junior et al. (2021)
TPC na admissão hospitalar vs. TPC mais ivermectina $100 \mathrm{mcg} / \mathrm{kg}$ ou $200 \mathrm{mcg} / \mathrm{kg}$ ou 400 $\mathrm{mcg} / \mathrm{kg}$.
Estudo piloto de ensaio aberto e randomizado
Brasil

\begin{tabular}{|c|c|c|c|c|}
\hline $\begin{array}{l}\text { Efeitos da ivermectina em } \\
\text { pacientes com COVID-19: } \\
\text { um ensaio clínico } \\
\text { multicêntrico, duplo-cego, } \\
\text { randomizado e controlado }\end{array}$ & $\begin{array}{c}\text { Shahbaznejad et } \\
\text { al. (2021) }\end{array}$ & $\begin{array}{l}\text { Ivermectina dose única }(15-24 \mathrm{~kg}=3 \mathrm{mg} ; 25- \\
30 \mathrm{~kg}=6 \mathrm{mg} ; 36-50 \mathrm{~kg}=9 \mathrm{mg} ; 51-80 \mathrm{~kg}=12 \mathrm{mg} ; \\
\text { e> } 80 \mathrm{~kg}=0,2 \mathrm{mg} / \mathrm{kg} \text { ) mais TP vs. TP } \\
\text { (hidroxicloroquina e/ou } \\
\text { lopinavir/ritonavir).Todos os participantes } \\
\text { receberam antibióticos e/ou oxigênio } \\
\text { suplementar conforme indicado. }\end{array}$ & $\begin{array}{l}\text { Ensaio clínico } \\
\text { multicêntrico, } \\
\text { duplo-cego, } \\
\text { randomizado e } \\
\text { controlado }\end{array}$ & Irã \\
\hline $\begin{array}{l}\text { Efeitos de uma dose única de } \\
\text { ivermectina nos resultados } \\
\text { clínicos e virais em } \\
\text { indivíduos infectados com } \\
\text { SARS-CoV-2 assintomáticos: } \\
\text { um ensaio clínico piloto no } \\
\text { Líbano }\end{array}$ & $\begin{array}{c}\text { Samaha et al. } \\
(2021)\end{array}$ & $\begin{array}{l}\text { Dose única de ivermectina de acordo com o } \\
\text { peso corporal }(45-64 \mathrm{~kg}=9 \mathrm{mg}, 65-84 \mathrm{~kg}= \\
12 \mathrm{mg} \text { ou }>85 \mathrm{~kg}=150 \mathrm{mcg} / \mathrm{kg}) \text { mais } \\
\text { suplementos profiláticos vs. suplementos } \\
\text { profiláticos como zinco }(30-50 \mathrm{mg} / \mathrm{dia}) \mathrm{e} \\
\text { vitamina C ( } 500 \mathrm{mg} \text { duas vezes ao dia). }\end{array}$ & $\begin{array}{c}\text { Ensaio clínico } \\
\text { randomizado }\end{array}$ & Líbano \\
\hline $\begin{array}{l}\text { Um curso de cinco dias de } \\
\text { ivermectina para o tratamento } \\
\text { da COVID-19 pode reduzir a } \\
\text { duração da doença }\end{array}$ & $\begin{array}{l}\text { Ahmed et al. } \\
\text { (2021) }\end{array}$ & $\begin{array}{l}\text { Ivermectina } 12 \mathrm{mg} \text { uma vez ao dia por } 5 \text { dias } \\
\text { vs. ivermectina } 12 \mathrm{mg} \text { em dose única mais } 200 \\
\text { mg de doxiciclina no dia } 1 \text {, seguido por } 100 \mathrm{mg} \\
\text { a cada } 12 \mathrm{~h} \text { durante os próximos } 4 \text { dias vs. } \\
\text { grupo de controle com placebo. }\end{array}$ & $\begin{array}{l}\text { Ensaio } \\
\text { randomizado, } \\
\text { duplo-cego e } \\
\text { controlado por } \\
\text { placebo }\end{array}$ & Bangladesh \\
\hline
\end{tabular}


Ivermectina em combinação tratamento dos sintomas de COVID-19: um ensaio randomizado com doxiciclina para $\mathrm{o}$

Dose única de ivermectina $12 \mathrm{mg}$ e doxiciclina $100 \mathrm{mg}$, duas vezes ao dia, por 5 dias mais TP (paracetamol, histamínicos, antitussígenos,
Mahmud et al. (2021) vitaminas, oxigenoterapia, heparina de baixo peso molecular, antibióticos, remdesivir, outros medicamentos antivirais e para condições comórbidas associadas, conforme necessidade) vs. placebo mais TP.
Ensaio

randomizado e Bangladesh cego
Nitazoxanida $500 \mathrm{mg} / 6$ horas, mais ribavirina $1200 \mathrm{mg}$ (doses divididas de $400 \mathrm{mg}$ ), mais ivermectina: $<60 \mathrm{~kg}$ ou $60-90 \mathrm{~kg}(200-$

Efeito da combinação de nitazoxanida, ribavirina e ivermectina mais suplemento de zinco (estudo

MANS.NRIZ) na depuração de COVID-19 leve
Elalfy et al. (2021) (dose fixa $30 \mathrm{mg}$ ) a cada $72 \mathrm{~h}$ até o final de 2 semanas de acordo com cada caso, mais zinco $300 \mu \mathrm{g} / \mathrm{kg}$ ), 3 tabletes de $6 \mathrm{mg}$ cada, $90-120 \mathrm{~kg}$ $(300-400 \mu \mathrm{g} / \mathrm{kg}), 4$ tabletes, $>120 \mathrm{~kg}, 5$ tabletes $30 \mathrm{mg}$ duas vezes ao dia vs. tratamento de suporte. controlado não

randomizado de

Egito

fase 1
Eficácia de uma terapia multifármaco consistindo de ivermectina, azitromicina, montelucaste e ácido acetilsalicílico para prevenir hospitalização e morte entre casos ambulatoriais de COVID-19 em Tlaxcala, México
Ivermectina $12 \mathrm{mg}$ em dose única, mais Azitromicina $500 \mathrm{mg}$ por 4 dias, mais

Lima-Morales et al. (2021).
Montelucaste $60 \mathrm{mg}$ no dia 1 , mais $10 \mathrm{mg}$ entre os dias 2 até 21, mais ácido acetilsalicílico $100 \mathrm{mg}$ por 30 dias vs. grupo controle (outro tipo de tratamento ou ainda nenhum tratamento).

Estudo $\quad$ México
comparativo

\section{ESTUDOS COM RESULTADOS NEGATIVOS PARA IVERMECTINA VS. COVID-19}

\begin{tabular}{|c|c|c|c|c|}
\hline $\begin{array}{c}\text { Ausência de eficácia de doses } \\
\text { padrão de ivermectina em } \\
\text { pacientes graves com } \\
\text { COVID19 }\end{array}$ & $\begin{array}{c}\text { Camprubí et al. } \\
\text { (2020) }\end{array}$ & $\begin{array}{l}\text { Ivermectina } 200 \mathrm{mcg} / \mathrm{kg} \text { em dose única mais } \\
\text { tratamento imunossupressor e procedimentos } \\
\text { hospitalares padrões vs. tratamento } \\
\text { imunossupressor, mas não recebendo } \\
\text { ivermectina. Todos os pacientes receberam } \\
\text { hidroxicloroquina e azitromicina e } \\
\text { lopinavir/ritonavir. Além disso, alguns } \\
\text { pacientes também receberam tocilizumabe, } \\
\text { esteroides, anakinra, siltuximab, remdesivir e } \\
\text { beta-interferon. }\end{array}$ & $\begin{array}{c}\text { Estudo } \\
\text { retrospectivo }\end{array}$ & Espanha \\
\hline
\end{tabular}

O efeito do tratamento

precoce com ivermectina na carga viral, sintomas e resposta humoral em pacientes com COVID-19 não grave: um ensaio clínico piloto, duplo-cego, controlado por placebo, randomizado
Ensaio piloto, randomizado, duplo-cego, Espanha controlado por placebo
Efeito da ivermectina no tempo de resolução dos sintomas entre adultos com COVID-19 leve. Um ensaio clínico randomizado
Chaccour et al. (2020). placebo.
Ivermectina $300 \mathrm{mcg} / \mathrm{kg}$ por dia durante 5 dias

Lopéz-Medina, et al. (2021) vs. placebo. Todavia, alguns pacientes em ambos os grupos, fizeram uso de outros medicamentos complementares.
Ensaio Clínico

Duplo-Cego

Randomizado
Colômbia 


\begin{tabular}{|c|c|c|c|c|}
\hline $\begin{array}{c}\text { Estudo randomizado de fase } 2 \\
\text { sobre cloroquina, } \\
\text { hidroxicloroquina ou } \\
\text { ivermectina em pacientes } \\
\text { hospitalizados com } \\
\text { manifestações graves de } \\
\text { infecção por SARS-CoV-2 }\end{array}$ & $\begin{array}{l}\text { Galan et al. } \\
(2021)\end{array}$ & $\begin{array}{l}\text { Sulfato de hidroxicloroquina ( } 400 \mathrm{mg} \text { duas } \\
\text { vezes no dia } 0 \text { e uma vez por dia do dia } 1 \text { ao dia } \\
\text { 4) vs. difostato de cloroquina ( } 450 \mathrm{mg} \text {, duas } \\
\text { vezes no dia } 0 \text { e uma vez ao dia a partir do dia } 1 \\
\text { ao dia } 4 \text { ) vs. ivermectina ( } 14 \mathrm{mg} \text { uma vez ao } \\
\text { dia, do dia } 0 \text { ao dia } 2+1 \text { comprimido de } \\
\text { placebo ao dia, no dia } 0,3 \text { e } 4 \text { ). Para } \\
\text { participantes com peso corporal inferior } 55 \mathrm{~kg} \text {, } \\
\text { a dose de ivermectina foi ajustada para } 10 \mathrm{mg} \\
\text { cada dose. }\end{array}$ & $\begin{array}{c}\text { Estudo } \\
\text { randomizado de } \\
\text { fase } 2\end{array}$ & Brasil \\
\hline $\begin{array}{l}\text { Estudo clínico avaliando a } \\
\text { eficácia da ivermectina no } \\
\text { tratamento da Covid-19: um } \\
\text { estudo randomizado } \\
\text { controlado }\end{array}$ & $\begin{array}{c}\text { Abd-Elsalam et } \\
\text { al. (2021) }\end{array}$ & $\begin{array}{l}\text { Ivermectina } 12 \mathrm{mg} / \text { dia por } 3 \text { dias, mais } \\
\text { protocolo de tratamento padrão vs. protocolo de } \\
\text { tratamento padrão que incluiu paracetamol, } \\
\text { oxigênio, antibiótico, oseltamivir e ventilação } \\
\text { mecânica invasiva com hidrocortisona, de } \\
\text { acordo com cada caso. }\end{array}$ & $\begin{array}{c}\text { Estudo } \\
\text { randomizado } \\
\text { controlado e } \\
\text { aberto }\end{array}$ & Egito \\
\hline $\begin{array}{l}\text { Risco de hospitalização para } \\
\text { pacientes ambulatoriais } \\
\text { Covid-19 tratados com vários } \\
\text { regimes de medicamentos no } \\
\text { Brasil: análise comparativa }\end{array}$ & $\begin{array}{l}\text { Fonseca et al. } \\
\qquad(2020)\end{array}$ & $\begin{array}{c}\text { Para pacientes que receberam alta: } \\
\text { hidroxicloroquina como primeira linha } \\
\text { tratamento ( } 400 \mathrm{mg} 2 \mathrm{X} / \text { dia no dia } 1,400 \\
\mathrm{mg} / \text { dia nos dias } 2-5) \text {, prednisona ( } 1 \mathrm{mg} / \mathrm{kg} / \mathrm{dia} \\
\text { por } 5 \mathrm{dias} \text {, máximo de } 80 \mathrm{mg} / \mathrm{dia}) \text {, azitromicina } \\
\text { (500 mg/dia por } 5 \text { dias), ivermectina ( } 12 \mathrm{mg} / \mathrm{dia} \\
\text { por } 2 \text { dias) mais medicamentos para alívio de } \\
\text { sintomas. Sulfato de zinco, oseltamivir e } \\
\text { nitazoxanida também foram disponíveis para } \\
\text { serem prescritos, mas eram usados com pouca } \\
\text { frequência. }\end{array}$ & $\begin{array}{c}\text { Análise } \\
\text { Comparativa }\end{array}$ & Brasil \\
\hline
\end{tabular}

Fonte: Autores (2021).

\section{Discussão}

Foram encontrados nas pesquisas nove estudos clínicos que evidenciaram benefícios da ivermectina em relação ao tratamento de pacientes positivados para o SARS-CoV-2. Em Bangladesh, um estudo retrospectivo, realizado por Khan et al. (2020) avaliou 248 pacientes com COVID-19, no qual 115 receberam ivermectina (uma dose de 12mg dentro de 24 horas após a admissão hospitalar) juntamente com tratamento padrão (TP) conforme necessário (antipiréticos, anti-histamínicos e antibióticos), enquanto 133 receberam apenas TP. No grupo tratado com ivermectina, verificou-se que nenhum dos pacientes desenvolveu patologia progressiva, como pneumonia ou complicações cardiovasculares, além de terem se tornado SARS-CoV2 negativos mais rapidamente (mediana 4 vs. 15 dias), possuírem estadias mais curtas no hospital (mediana 9 vs. 15 dias), e taxas de mortalidade menores $(0,9 \%$ vs. 6,8\%). Além disso, menos pacientes desse grupo necessitaram de inalação de oxigênio ( $9,6 \%$ vs. $45,9 \%)$, desenvolveram desconforto respiratório (2,6\% vs. 15,8\%), necessitaram de tratamento com antibióticos $(15,7 \%$ vs. $60,2 \%)$ e gestão de terapia intensiva ( $0,9 \%$ vs. $8,3 \%)$.

Corroborando com esses achados, o estudo de coorte retrospectivo de Rajter et al. (2021), também evidenciaram taxas de mortalidades menores no grupo tratado com ivermectina, especialmente em pacientes com envolvimento pulmonar grave, em um estudo que avaliou 173 pacientes que receberam pelo menos uma dose oral de ivermectina (200mcg/kg), podendo ser administrada a critério do médico uma segunda dose no $7^{\circ}$ dia de tratamento, e 107 pacientes que receberam tratamento usual (sem ivermectina). Todavia, a maioria dos pacientes em ambos os grupos também recebeu corticosteroides, hidroxicloroquina e/ou azitromicina. Portanto, ainda que não tenham sido encontradas diferenças estatisticamente significativas nas taxas de 
extubação ou tempo de permanência no hospital, as taxas de mortalidade apresentaram-se reduzidas no grupo que recebeu ivermectina, evidenciando benefício do seu uso frente a esse parâmetro.

Pott-Junior et al. (2021), conduziram um estudo piloto de ensaio aberto e randomizado, no Brasil, para avaliar os efeitos antivirais e a segurança de várias doses de ivermectina em pacientes com sintomas clínicos leve de COVID-19. Para tal, avaliou-se 31 pacientes, que receberam tratamento padrão de cuidados (TPC) na admissão hospitalar ( $\mathrm{n}=4$ ), TPC mais ivermectina $100 \mathrm{mcg} / \mathrm{kg}(\mathrm{n}=6), 200 \mathrm{mcg} / \mathrm{kg}(\mathrm{n}=14)$ ou $400 \mathrm{mcg} / \mathrm{kg}(\mathrm{n}=7)$. Vale ressaltar que apenas alguns pacientes receberam glicocorticoides, antibióticos e heparina durante a hospitalização. Verificou-se que todos os pacientes exibiram uma redução na carga viral da SARS-CoV-2 em 7 dias. Todavia, aqueles que receberam TPC mais ivermectina (especialmente nas doses de 200 e $400 \mathrm{mcg} / \mathrm{kg}$ ) apresentaram um tempo menor para a obtenção de dois testes consecutivos de RT PCR para SARS-CoV-2 negativos.

Um ensaio clínico duplo-cego randomizado realizado no Irã, avaliou 35 pacientes receberam dose única de ivermectina de acordo com o peso corporal $(15-24 \mathrm{~kg}=3 \mathrm{mg} ; 25-30 \mathrm{~kg}=6 \mathrm{mg} ; 36-50 \mathrm{~kg}=9 \mathrm{mg} ; 51-80 \mathrm{~kg}=12 \mathrm{mg} ; \mathrm{e}>80 \mathrm{~kg}$ $=0,2 \mathrm{mg} / \mathrm{kg}$ ) mais TP e 34 pacientes que receberam TP, que incluiu hidroxicloroquina e/ou lopinavir/ritonavir no grupo controle. Além disso, todos os participantes receberam antibióticos apropriados e/ou oxigênio suplementar conforme indicado. Os resultados mostraram que a duração dos sintomas clínicos como dispneia $(2,4$ vs. 3,7 dias $)$ e tosse $(3,1$ vs. 4,8) foram menores no grupo ivermectina do que no grupo controle, respectivamente. Além disso, os tempos médios de internação hospitalar também foram menores no grupo ivermectina (6,9 vs. 8,3 dias) e a frequência de linfopenia diminuiu para 14,3\% no grupo de ivermectina e não mudou no grupo de controle. Ademais, não foram registrados eventos adversos potenciais e graves em ambos os grupos (Shahbaznejad et al., 2021).

No Líbano, Samaha et al. (2021) realizaram um ensaio clínico randomizado para avaliar a eficácia da ivermectina na produção de desfechos clínicos favoráveis em indivíduos assintomáticos positivos para SARS-CoV-2. Para tal, 50 indivíduos foram incluídos no grupo controle recebendo suplementos profiláticos recomendados no Líbano, como zinco (30-50 mg/dia) e vitamina C (500 mg duas vezes ao dia) e 50 indivíduos receberam dose única de ivermectina de acordo com o peso corporal $(45-64 \mathrm{~kg}=9 \mathrm{mg}, 65-84 \mathrm{~kg}=12 \mathrm{mg}$ ou $>85 \mathrm{~kg}=150 \mathrm{mcg} / \mathrm{kg})$, além dos mesmos suplementos profiláticos do grupo controle. A presença viral entre os dois grupos foi medida usando o indicador de limiar de ciclo (valores $\mathrm{Ct}$ ), que fornece uma medida relativa da quantidade de vírus na amostra, sendo que um valor $\mathrm{Ct}$ de $\geq 30$ foi considerado indicativo de cargas virais insignificantes ou um vírus não viável. No dia zero ambos os grupos possuíam valores semelhantes de $\mathrm{Ct}$, ou seja, cargas virais semelhantes. Após 72 horas do início do tratamento, a ivermectina aumentou significativamente o valor Ct de 15,13 $\pm 2,07$ a

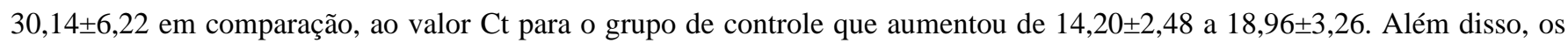
resultados mostraram que os indivíduos que receberam ivermectina desenvolveram menos sintomas de febre, anosmia, mialgia e perda do paladar em comparação com o grupo de controle, e também não tiveram nenhum caso de hospitalização, contra três casos do grupo controle. Dessa forma, a ivermectina mostrou ser eficaz em fornecer benefícios clínicos em tratamento de pacientes assintomáticos positivos para SARS-CoV-2.

Em um ensaio randomizado, duplo-cego e controlado por placebo, conduzido por Ahmed et al. (2021), 72 pacientes hospitalizados foram tratados com ivermectina oral isoladamente (12 $\mathrm{mg}$ uma vez ao dia por 5 dias), ou ivermectina oral em combinação com doxiciclina (12 mg de ivermectina em dose única e $200 \mathrm{mg}$ de doxiciclina no dia 1, seguido por $100 \mathrm{mg}$ a cada 12h durante os próximos 4 dias), e um grupo de controle com placebo, verificou que a eliminação do vírus foi mais rápida (avaliada através do rRT-PCR) apenas no grupo que recebeu ivermectina por cinco dias. Todavia, ainda que o trabalho encontrou benefícios do uso da ivermectina frente ao tempo da eliminação viral, não foram encontradas diferenças estatisticamente significativas para remissão da febre, tosse ou dor de garganta nos que receberam os tratamentos em comparação ao placebo. 
Corroborando com esses estudos, Mahmud et al. (2021) avaliaram a combinação da ivermectina com doxiciclina para reduzir o tempo de recuperação clínica em pacientes com COVID-19 leve a moderado. Foram analisados 183 pacientes que receberam dose única de ivermectina $12 \mathrm{mg}$ e doxiciclina $100 \mathrm{mg}$, duas vezes ao dia, por 5 dias, além de TP, que incluiu paracetamol, histamínicos, antitussígenos, vitaminas, oxigenoterapia, heparina de baixo peso molecular, antibióticos, remdesivir, outros medicamentos antivirais e para condições comórbidas associadas, conforme indicação e necessidade e 180 pacientes que receberam placebo mais TP. Os resultados mostraram que o período de recuperação médio foi de 7 dias no grupo ivermectina e 9 dias no grupo placebo, além disso a proporção de pacientes que se recuperaram em 7 dias do início do tratamento foi maior no grupo ivermectina do que no grupo placebo (61\% vs. 44\%). Ademais, a probabilidade de apresentar sintomas que persistiram por mais de 12 dias foi menor no grupo ivermectina (42 [23\%] vs. 67 [37\%]) e a proporção de pacientes que permaneceram RT-PCR positivos no dia 14 e cuja doença não progrediu também foi menor no grupo ivermectina, o que mostra benefícios da combinação desses dois fármacos para o tratamento de alguns parâmetros da COVID19.

Um ensaio controlado não randomizado de fase 1, de Elalfy et al. (2021) realizado no Egito, verificou que a taxa e o tempo de eliminação viral em indivíduos com COVID-19, foram menores no grupo de pacientes que receberam uma combinação de nitazoxanida, ribavirina e ivermectina mais zinco (62 pacientes) em comparação com aqueles que receberam tratamento de suporte (51 pacientes), que incluiu tratamento sintomático com paracetamol, zinco, boa nutrição e hidratação, além do uso da azitromicina sendo avaliado caso a caso. As taxas de depuração cumulativas, ou seja, a eliminação viral, no $15^{\circ}$ dia foram de $13,7 \%$ e $88,7 \%$ no tratamento de suporte e nos grupos antivirais combinados respectivamente, evidenciando menor tempo para a eliminação viral, no grupo tratado com a terapia medicamentosa em estudo. Além disso, os efeitos colaterais mais comuns para a terapia antiviral combinada foram distúrbios do trato gastrointestinal, urina colorida e palpitações.

Além disso, Lima-Morales et al. (2021) avaliaram a eficácia de uma terapia multifármaco consistindo de ivermectina, azitromicina, montelucaste e ácido acetilsalicílico, denominada TNR4, para prevenir hospitalização e morte entre casos ambulatoriais de COVID-19 em Tlaxcala, México. Comparou-se 481 casos receberam a terapia TNR4, enquanto 287 receberam outro tratamento, como o uso de AINEs, ou antibiótico combinado com AINEs ou corticosteroides, ou antivirais junto com AINEs ou corticosteroides, ou ainda nenhum medicamento. Os resultados mostraram que a probabilidade de recuperação, riscos de hospitalização e morte foram menores no grupo TNR4 do que no grupo comparativo. A probabilidade de recuperação em 14 dias foi 3,4 vezes maior nos pacientes tratados com a terapia TNR4, uma vez que 85\% dos casos desse grupo se recuperaram nesse tempo, enquanto obteve-se recuperação em apenas $59 \%$ dos casos no grupo de comparação. Além disso, os pacientes que receberam TNR4 tiveram um risco $75 \%$ menor de serem hospitalizados e $81 \%$ menor de morrer do que o grupo em comparação.

Em contrapartida, a pesquisa também evidenciou seis estudos não favoráveis para o emprego da ivermectina como tratamento para a COVID-19. Um estudo realizado em Barcelona, na Espanha, avaliou um grupo de 26 pacientes tratados com terapia imunossupressora, sendo que os 13 pacientes tratados com dose única de ivermectina $200 \mathrm{mcg} / \mathrm{kg}$, não obtiveram melhora nos resultados clínicos e microbiológicos decorrentes de um quadro grave de COVID-19, em comparação aos 13 pacientes do grupo controle. O exame de PCR realizado entre 3 e 5 dias após o recebimento da ivermectina, se deu positivo em cinco pacientes do grupo ivermectina e em quatro pacientes no grupo controle. Além disso, foi observada entre oito a onze dias após o tratamento, uma notável melhora clínica em nove participantes que receberam ivermectina e em dez participantes do grupo de comparação, demonstrando que independente do tratamento com a ivermectina, os pacientes evoluíram bem da infecção. Vale ressaltar que alguns pacientes também receberam outros medicamentos como tocilizumabe, esteroides, anakinra, siltuximab, remdesivir e beta-interferon. Ademais, seguindo os protocolos do hospital no momento do estudo, todos 
os pacientes receberam hidroxicloroquina e azitromicina, e com exceção de um paciente do grupo ivermectina, todos também foram tratados com lopinavir/ritonavir (Camprubí et al., 2020).

Um ensaio piloto, randomizado, duplo-cego, controlado por placebo realizado em Navarra, na Espanha, por Chaccour et al. (2020) verificou que não houve diferença na proporção de pacientes positivos para PCR no dia 7 pós-tratamento, e na diminuição das cargas virais nos dias 4 e 7 pós-tratamento, bem como, títulos de IgG mais baixos no dia 21 pós-tratamento, na comparação dos pacientes que receberam ivermectina $400 \mathrm{mcg} / \mathrm{kg}$, dose única $(\mathrm{n}=12)$ ou placebo $(\mathrm{n}=12)$. Não houve grandes diferenças entre o grupo ivermectina e placebo no que diz respeito a presença de febre, mal-estar geral, dor de cabeça ou congestão nasal, todavia, verificou-se que os pacientes no grupo da ivermectina se recuperaram mais cedo da hiposmia/anosmia.

Ademais, Lopéz-Medina et al. (2021) também realizaram um ensaio clínico duplo-cego randomizado, cujos pacientes receberam ivermectina $300 \mathrm{mcg} / \mathrm{kg}$ por dia durante 5 dias $(\mathrm{n}=200)$ ou placebo $(\mathrm{n}=200)$, para avaliar a resolução dos sintomas em um período de acompanhamento de 21 dias. Todavia, alguns pacientes em ambos os grupos, fizeram uso de outros medicamentos, como anti-inflamatórios não esteroides, antibióticos, antipiréticos, glicocorticoides, anticoagulantes, interferon, colchicina, aciclovir, antidiarreicos, antieméticos, anti-histamínicos, antiparasitários, antiespasmódicos, antitussígenos, medicamentos naturais ou homeopáticos, inibidores da bomba de próton e salbutamol. Assim sendo, verificou-se que a ivermectina não mostrou diminuir o tempo de resolução de sintomas, uma vez que o tempo médio para resolução foi de 10 dias no grupo ivermectina em comparação com 12 dias no grupo placebo. No dia $21,82 \%$ dos pacientes do grupo de ivermectina e $79 \%$ no grupo de placebo não apresentavam mais sintomas. Os eventos adversos mais mencionados pelos pacientes foram cefaleia, tontura e diarreia, com números de pacientes bem parecidos em ambos os grupos.

Galan et al. (2021) conduziram um estudo duplo-cego randomizado de fase 2, para avaliar pacientes internados em um hospital de Roraima, no Brasil, que foram randomizados em uma razão 1: 1: 1, para o tratamento com sulfato de hidroxicloroquina $400 \mathrm{mg}$ duas vezes no dia 0 e uma vez por dia do dia 1 ao dia 4 ( $\mathrm{n}=54$ ) ou difostato de cloroquina $450 \mathrm{mg}$, duas vezes no dia 0 e uma vez ao dia a partir do dia 1 ao dia $4(\mathrm{n}=61)$ ou ivermectina $14 \mathrm{mg}$ uma vez ao dia, do dia 0 ao dia 2 +1 comprimido de placebo ao dia, no dia 0, 3 e 4. Para participantes com peso corporal inferior $55 \mathrm{~kg}$, a dose de ivermectina foi ajustada para $10 \mathrm{mg}$ cada dose $(\mathrm{n}=53)$. Além disso, de acordo com o protocolo do hospital, alguns pacientes receberam corticoides, a critério médico, e doses profiláticas de enoxaparina, bem como, azitromicina e ceftrixona em caso de síndrome do desconforto respiratório agudo e oseltamivir para suspeita de infecção por influenza. Verificou-se que esses fármacos não reduziram a necessidade de oxigênio suplementar, admissão em UTI, ventilação invasiva ou morte, em pacientes hospitalizados com uma forma grave de COVID-19, sugerindo inefetividade dos medicamentos.

Um estudo randomizado aberto e controlado realizado no Egito, avaliou 164 pacientes com quadro clínico de COVID19 de leve a moderado, dos quais 82 pacientes receberam ivermectina (12 $\mathrm{mg} /$ dia por 3 dias) e protocolo padrão de tratamento de acordo com o protocolo do Ministério da Saúde egípcio para o tratamento da COVID-19, e 82 pacientes receberam somente o protocolo padrão de tratamento por 14 dias, que incluiu paracetamol, oxigênio, antibiótico, oseltamivir e ventilação mecânica invasiva com hidrocortisona, de acordo com cada caso. Os resultados mostraram que não houve qualquer diferença estatisticamente significativa nos desfechos analisados. Em relação a mortalidade, três pacientes no grupo ivermectina e quatro pacientes do grupo controle evoluíram a óbito, três pacientes de cada grupo precisaram de ventilação mecânica e apesar do grupo ivermectina apresentar um tempo de internação hospitalar mais curto $(8,82 \pm 4,94$ dias) do que o grupo controle $(10,97 \pm$ 5,28 dias), isso também não foi estatisticamente significativo, indicando a ineficácia da ivermectina para o tratamento da COVID-19 (Abd-Elsalam et al., 2021).

Por fim, uma análise comparativa realizada no Brasil, feita por Fonseca et al. (2020) avaliou os riscos de hospitalização subsequente, em 717 pacientes com mais de 40 anos, que deram entrada em uma rede ambulatorial de prontos- 
socorros com sintomas de influenza e que receberam a critério médico, tratamento precoce para a COVID-19, que incluiu medicamentos como a hidroxicloroquina, azitromicina, ivermectina, oseltamivir, sulfato de zinco, nitazoxanida e prednisona (a última começando no dia 6 dos sintomas). Apesar do uso da hidroxicloroquina, prednisona ou ambos terem reduzido significativamente o risco de hospitalização em 50 - 60\%, o uso da ivermectina, bem como, da azitromicina e oseltamivir não reduziram substancialmente esse risco.

Todavia, vale ressaltar que a maioria dos estudos possuem uma série de limitações que implicam na falta de uma compreensão clara e uniforme do efeito da ivermectina em pacientes com COVID-19. Dentre as limitações encontradas, o pequeno tamanho das amostras se fez presente em vários estudos, implicando em uma possível não representatividade de uma população e dificultando o desempenho dos testes estatísticos.

Além disso, outra limitação está associada ao momento em que a intervenção foi avaliada, além de algumas literaturas evidenciarem que os antivirais exercem maior eficácia clínica quando administrados precocemente, os estudos aqui analisados divergiram nesse aspecto, sendo alguns estudos administraram a ivermectina mais precocemente, enquanto outros em estágios mais tardios da doença, o que dificulta a comparação da ação do fármaco entre esses estudos. Ademais, a maioria dos estudos utiliza a dose eficaz antiparasitária para a ivermectina $(200 \mu \mathrm{g} / \mathrm{kg}$ de peso corporal), que não é a dose encontrada do estudo in vitro de Caly et al. (2020) para SARS-CoV-2, por exemplo.

Todavia, outro parâmetro importante diz respeito a gravidade da doença, uma vez que alguns estudos trabalharam com quadros leves da COVID-19, enquanto outros com casos moderados e graves. Observou-se que alguns estudos deixam a desejar quanto a informação de quando exatamente a ivermectina é administrada após o teste positivo para SARS-CoV-2. Tais fatores são importantes para entender se o efeito do medicamento depende do tempo e da gravidade dos sintomas. Vale ressaltar, que o diagnóstico da doença, em alguns casos, não pôde ser confirmado por PCR em todos os pacientes, e que foram levados em consideração apenas sintomas, sinais e alterações em exames laboratoriais.

Ainda assim, é possível citar a falta de clareza na metodologia de alguns estudos, principalmente no que diz respeito ao tratamento padrão, complementar à administração da ivermectina, utilizado nos trabalhos. Ao mesmo passo que muitos não informaram quais eram os medicamentos utilizados no tratamento padrão, outros utilizaram diferentes medicamentos em pacientes de um mesmo grupo (por exemplo, de todos que receberam ivermectina, alguns pacientes também receberam medicamentos complementares que divergiram entre os integrantes desse grupo) ou de grupos diferentes (por exemplo, pacientes do grupo controle receberam alguns medicamentos complementares diferentes do grupo ivermectina). Além disso, a maioria dos pacientes estavam internados e recebiam tratamento consistindo na utilização de vários fármacos, o que pode ter interferido para permitir a avaliação do uso da ivermectina frente a COVID-19.

Nesse sentido, entende-se que os estudos apresentam uma grande variedade em relação ao momento da administração da ivermectina, além da gravidade no quadro clínico dos pacientes e nos tratamentos complementares utilizados. A COVID-19 ainda é considerada uma doença nova, possuindo informações e estudos científicos conflitantes para embasar as condutas e as pesquisas. Por conta disso, não existe ainda um protocolo de tratamento ou um padrão ouro para essa doença. Ademais, vale ressaltar que a sintomatologia da doença é inespecífica e que a morbidade de cada paciente influencia na terapêutica utilizada (Xavier et al., 2020).

A ivermectina em geral, é bem tolerada e segura para os seres humanos. No entanto, seu uso deve ser feito de forma racional e cautelosa, sob orientação de profissionais habilitados, visto que podem ocorrer eventos adversos neurológicos relacionadas ao seu uso, como confusão, tremores, convulsão, inchaço local, e vômitos (Molento, 2020). Além disso, as reações adversas mais comuns incluem diarreia, náusea, dor abdominal e constipação, podendo também ocorrer tontura, sonolência, vertigem, coceira, lesão de pele e urticária, com menor frequência. Deve-se também, tomar cuidado com a administração da ivermectina em associação com outros fármacos potencializadores da atividade GABA, uma vez que ela pode 
interagir com os receptores GABA no Sistema Nervoso Central. Ademais, a ivermectina não deve ser utilizada por gestantes e sua segurança não é comprovada em crianças menores de 5 anos ou com menos de $15 \mathrm{~kg}$ (Abbott, 2021).

Segundo o Ministério da Saúde (2021), a dose mínima efetiva da ivermectina é de 6mg, e a máxima efetiva é de 200 $\mathrm{mcg} / \mathrm{kg}$, ambos em dose única. Todavia, tomar doses excessivas desse medicamento é perigoso e pode causar sérios danos. A overdose por ivermectina pode causar náusea, vômito, diarreia, hipertensão, reações alérgicas, tontura, ataxia, convulsão, coma e até morte (Food and Drug Administration [FDA], 2021).

Durante a pandemia, foi possível verificar no Brasil a ampla disseminação da ideia do uso do "kit-covid" que incluía a ivermectina como medida de profilaxia preventiva e tratamento para a COVID-19, tanto por pessoas não autorizadas através de redes sociais, quanto por profissionais médicos, autoridades públicas e em algumas páginas oficiais de órgãos de saúde, sem requisitos mínimos científicos de segurança, eficácia ou efetividade. Como resultado, observou-se um aumento na prática da automedicação e um estímulo coletivo ao uso irracional desse medicamento, impulsionada pela mídia, autoridades e pela população em geral. As vendas da ivermectina passaram de $\mathrm{R}$ \$ 44 milhões em 2019 para $\mathrm{R} \$ 409$ milhões em 2020 (Melo, et al., 2021; Molento, 2020).

As organizações e órgãos mundiais de saúde, possuem posicionamentos semelhantes em relação ao tratamento da COVID-19 e o uso da ivermectina. A OMS e a Organização Pan-Americana da Saúde (OPAS) não recomendam o uso da ivermectina para o tratamento da COVID-19, uma vez que as revisões feitas dos estudos realizados em humanos evidenciaram incerteza nos benefícios e danos potenciais do uso desse fármaco. Embora estimativas sugiram benefícios com ivermectina, fatores como limitações metodológicas dos estudos apontam que as evidências são insuficientes, fato esse que foi reforçado pelo presente trabalho, e que dessa forma a ivermectina pode não reduzir significativamente a mortalidade e provavelmente não melhora o tempo de resolução dos sintomas (Pan American Health Organization [PAHO], 2021).

Os Institutos Nacionais da Saúde (NIH - National Institute of Health), agência governamental do Departamento de Saúde e Serviços Humanos dos Estados Unidos, impõe que não há dados suficientes para recomendar ou não o uso da ivermectina para o tratamento da COVID-19. Sendo assim, são necessários resultados de ensaios clínicos bem desenhados e conduzidos para fornecer evidências mais conclusivas a respeito do tema. Eles ainda evidenciam que hoje o único tratamento aprovado pelo FDA para o tratamento da COVID-19 é o Remdesivir (National Institute of Health [NIH], 2021). Por sua vez, a Agência Europeia de Medicamentos (EMA), também validou o Remdesivir como único tratamento autorizado para a COVID19 na União Europeia (European Medicines Agency [EMA], 2021).

\section{Conclusão}

Diante do exposto, verifica-se que apesar de alguns estudos mostrarem benefícios do uso da ivermectina para alguns parâmetros da COVID-19, outros pesquisadores colocam essa questão em controvérsia quando evidenciam que a ivermectina é inefetiva para o tratamento da infecção. Todavia, a maioria dessas pesquisas possuem uma série de limitações metodológicas, que dificultam a análise dos dados, interpretação dos resultados dos trabalhos, além de dificultar comparações entre eles. Dessa forma, a evidência atual sobre o uso desse medicamento para tratar pacientes com COVID-19 é inconclusiva.

Assim sendo, existe a necessidade do avanço das pesquisas, através de ensaios clínicos adequados e bem estruturados, para confirmar ou descartar essas descobertas, e fornecer orientações mais específicas e baseadas em evidências científicas sobre o papel da ivermectina no tratamento da COVID-19.

Dessa forma, futuros trabalhos podem abordar os novos estudos clínicos que ainda serão realizados ou que já estão em andamento, para analisar seus resultados, metodologia e limitações, de modo a contribuir ainda mais para fomentar de forma científica considerações plausíveis e confiáveis a respeito desse tema, servindo de subsídio para a população e a comunidade 
científica. Ademais, estudos de revisão integrativa com outros fármacos empregados no tratamento da COVID-19 também são importantes para melhorar o prognóstico da doença.

\section{Referências}

Abbott (2021). Bula para o profissional de saúde. Revectina. Recuperado de https://dam.abbott.com/pt-br/documents/pdfs/nossas-bulas/r/BU-16-Revectinabula-profissional-final.pdf

Abd-Elsalam, S., Noor, R. A., Badawi, R., Khalaf, M., Esmail, E. S., Soliman, S., Abd El Ghafar, M. S., Elbahnasawy, M., Moustafa, E. F., Hassany, S. M., Medhat, M. A., Ramadan, H. K., Eldeen, M., Alboraie, M., Cordie, A., \& Esmat, G. (2021). Clinical study evaluating the efficacy of ivermectin in COVID-19 treatment: A randomized controlled study. Journal of medical virology, 93(10), 5833-5838. https://doi.org/10.1002/jmv.27122

Ahmed, S., Karim, M. M., Ross, A. G., Hossain, M. S., Clemens, J. D., Sumiya, M. K., Phru, C. S., Rahman, M., Zaman, K., Somani, J., Yasmin, R., Hasnat, M. A., Kabir, A., Aziz, A. B., \& Khan, W. A. (2021). A five-day course of ivermectin for the treatment of COVID-19 may reduce the duration of illness. International journal of infectious, 103, 214-216. https://doi.org/10.1016/j.ijid.2020.11.191

Caly, L., Druce, J. D., Catton, M. G., Jans, D. A., \& Wagstaff, K. M. (2020). The FDA-approved drug ivermectin inhibits the replication of SARS-CoV-2 in vitro. Antiviral research, 178, 104787. https://doi.org/10.1016/j.antiviral.2020.104787

Chaccour, C., Casellas, A., Blanco-Di Matteo, A., Pineda, I., Fernandez-Montero, A., Ruiz-Castillo, P., Richardson, M. A., Rodríguez-Mateos, M., JordánIborra, C., Brew, J., Carmona-Torre, F., Giráldez, M., Laso, E., Gabaldón-Figueira, J. C., Dobaño, C., Moncunill, G., Yuste, J. R., Del Pozo, J. L., Rabinovich, N. R., Schöning, V., ... Fernández-Alonso, M. (2021). The effect of early treatment with ivermectin on viral load, symptoms and humoral response in patients with non-severe COVID-19: A pilot, double-blind, placebo-controlled, randomized clinical trial. EClinicalMedicine, 32, 100720. https://doi.org/10.1016/j.eclinm.2020.100720

Camprubí, D., Almuedo-Riera, A., Martí-Soler, H., Soriano, A., Hurtado, J. C., Subirà, C., Grau-Pujol, B., Krolewiecki, A., \& Muñoz, J. (2020). Lack of efficacy of standard doses of ivermectin in severe COVID-19 patients. PloS one, 15(11), e0242184. https://doi.org/10.1371/journal.pone.0242184

Dos Santos W. G. (2020). Natural history of COVID-19 and current knowledge on treatment therapeutic options. Biomedicine \& pharmacotherapy = Biomedecine \& pharmacotherapie, 129, 110493. https://doi.org/10.1016/j.biopha.2020.110493

Elalfy, H., Besheer, T., El-Mesery, A., El-Gilany, A. H., Soliman, M. A., Alhawarey, A., Alegezy, M., Elhadidy, T., Hewidy, A. A., Zaghloul, H., Neamatallah, M., Raafat, D., El-Emshaty, W. M., Abo El Kheir, N. Y., \& El-Bendary, M. (2021). Effect of a combination of nitazoxanide, ribavirin, and ivermectin plus zinc supplement (MANS.NRIZ study) on the clearance of mild COVID-19. Journal of medical virology, 93(5), 3176-3183. https://doi.org/10.1002/jmv.26880

European Medicines Agency. (2021). COVID-19 treatments: authorised. Recuperado de https://www.ema.europa.eu/en/human-regulatory/overview/publichealth-threats/coronavirus-disease-covid-19/treatments-vaccines/treatments-covid-19/covid-19-treatments-authorised

Fonseca, S. N. S., de Queiroz Sousa, A., Wolkoff, A. G., Moreira, M. S., Pinto, B. C., Valente Takeda, C. F., Rebouças, E., Vasconcellos Abdon, A. P., Nascimento, A., \& Risch, H. A. (2020). Risk of hospitalization for Covid-19 outpatients treated with various drug regimens in Brazil: Comparative analysis. Travel medicine and infectious disease, 38, 101906. https://doi.org/10.1016/j.tmaid.2020.101906

Food and Drug Administration. (2021). Why You Should Not Use Ivermectin to Treat or Prevent COVID-19. Recuperado de https://www.fda.gov/consumers/consumer-updates/why-you-should-not-use-ivermectin-treat-or-prevent-covid-19

Galan, L., Santos, N., Asato, M. S., Araújo, J. V., de Lima Moreira, A., Araújo, A., Paiva, A., Portella, D., Marques, F., Silva, G., de Sousa Resende, J., Tizolim, M. R., Santos, P. L., Buttenbender, S. F., de Andrade, S. B., Carbonell, R., Da Rocha, J. G., de Souza, R., \& da Fonseca, A. J. (2021). Phase 2 randomized study on chloroquine, hydroxychloroquine or ivermectin in hospitalized patients with severe manifestations of SARS-CoV-2 infection. Pathogens and global health, 115(4), 235-242. https://doi.org/10.1080/20477724.2021.1890887

Guo, Y. R., Cao, Q. D., Hong, Z. S., Tan, Y. Y., Chen, S. D., Jin, H. J., Tan, K. S., Wang, D. Y., \& Yan, Y. (2020). The origin, transmission and clinical therapies on coronavirus disease 2019 (COVID-19) outbreak - an update on the status. Military Medical Research, 7(1), 11. https://doi.org/10.1186/s40779020-00240-0

Hamed, M. A. (2020). An overview on COVID-19: reality and expectation. Bull Natl Res Cent. 44(1):86. doi: 10.1186/s42269-020-00341-9.

Heidary, F., \& Gharebaghi, R. (2020) Ivermectin: a systematic review from antiviral effects to COVID-19 complementary regimen. The Journal of Antibiotics 73, 593-602. https://doi.org/10.1038/s41429-020-0336-Z

Jans, D. A., \& Wagstaff, K. M. (2020). Ivermectin as a Broad-Spectrum Host-Directed Antiviral: The Real Deal?. Cells, 9(9), 2100. https://doi.org/10.3390/cells9092100

Kaur, H., Shekhar, N., Sharma, S., Sarma, P., Prakash, A., \& Medhi, B. (2021). Ivermectin as a potential drug for treatment of COVID-19: an in-sync review with clinical and computational attributes. Pharmacological reports : PR, 73(3), 736-749. https://doi.org/10.1007/s43440-020-00195-y

Khan, M., Khan, M., Debnath, C. R., Nath, P. N., Mahtab, M. A., Nabeka, H., Matsuda, S., \& Akbar, S. (2020). Ivermectin Treatment May Improve the Prognosis of Patients With COVID-19. Archivos de bronconeumologia, 56(12), 828-830. https://doi.org/10.1016/j.arbres.2020.08.007

Köche, J. C. (2011). Fundamentos de metodologia científica: teoria da ciência e iniciação à pesquisa. Petrópolis, RJ: Vozes.

Li, N., Zhao, L., \& Zhan, X. (2020). Quantitative proteomics reveals a broad-spectrum antiviral property of ivermectin, benefiting for COVID-19 treatment. Cell Physiol, 236, 2959-2975. https://doi.org/10.1002/jcp.30055 
Lima-Morales, R., Méndez-Hernández, P., Flores, Y. N., Osorno-Romero, P., Sancho-Hernández, C. R., Cuecuecha-Rugerio, E., Nava-Zamora, A., Hernández-Galdamez, D. R., Romo-Dueñas, D. K., \& Salmerón, J. (2021). Effectiveness of a multidrug therapy consisting of Ivermectin, Azithromycin, Montelukast, and Acetylsalicylic acid to prevent hospitalization and death among ambulatory COVID-19 cases in Tlaxcala, Mexico. International journal of infectious diseases, 105, 598-605. https://doi.org/10.1016/j.ijid.2021.02.014

Lima, W. G., Brito, J., Overhage, J., \& Nizer, W. (2020). The potential of drug repositioning as a short-term strategy for the control and treatment of COVID19 (SARS-CoV-2): a systematic review. Archives of virology, 165(8), 1729-1737. https://doi.org/10.1007/s00705-020-04693-5

López-Medina, E., López, P., Hurtado, I. C., Dávalos, D. M., Ramirez, O., Martínez, E., Díazgranados, J. A., Oñate, J. M., Chavarriaga, H., Herrera, S., Parra, B., Libreros, G., Jaramillo, R., Avendaño, A. C., Toro, D. F., Torres, M., Lesmes, M. C., Rios, C. A., \& Caicedo, I. (2021). Effect of Ivermectin on Time to Resolution of Symptoms Among Adults With Mild COVID-19: A Randomized Clinical Trial. JAMA, 325(14), 1426-1435. https://doi.org/10.1001/jama.2021.3071

Mahmud, R., Rahman, M. M., Alam, I., Ahmed, K., Kabir, A., Sayeed, S., Rassel, M. A., Monayem, F. B., Islam, M. S., Islam, M. M., Barshan, A. D., Hoque, M. M., Mallik, M. U., Yusuf, M. A., \& Hossain, M. Z. (2021). Ivermectin in combination with doxycycline for treating COVID-19 symptoms: a randomized trial. The Journal of international medical research, 49(5). https://doi.org/10.1177/03000605211013550

Melo, J. R. R.; Duarte, E. C.; Moraes, M. V.; Fleck, K. \& Arrais, P. S. D. (2021). Automedicação e uso indiscriminado de medicamentos durante a pandemia da COVID-19. Cad. Saúde Pública 37(4). doi: 10.1590/0102-311X00053221

Mendes, K. D. S., Silveira, R. C. de C. P., \& Galvão, C. M. (2019). Uso De Gerenciador De Referências Bibliográficas Na Seleção Dos Estudos Primários Em Revisão Integrativa. Texto \& Contexto - Enfermagem, 28. https://doi.org/10.1590/1980-265x-tce-2017-0204

Ministério da Saúde. (2021). Ivermectina para o tratamento de pacientes com COVID-19. Recuperado de https://www.gov.br/saude/ptbr/coronavirus/publicacoes-tecnicas/notas-tecnicas/nota-tecnica-ivermectina-covid-19

Mishra, S. K., \& Tripathi, T. (2021). One year update on the COVID-19 pandemic: Where are we now?. Acta tropica,214, 105778. https://doi.org/10.1016/j.actatropica.2020.105778

Molento M. B. (2020). COVID-19 and the rush for self-medication and self-dosing with ivermectin: A word of caution. One health (Amsterdam, Netherlands), 10, 100148. https://doi.org/10.1016/j.onehlt.2020.100148

National Institute of Health. (2021). Antiviral Drugs That Are Approved or Under Evaluation for the Treatment of COVID-19. Recuperado de https://www.covid19treatmentguidelines.nih.gov/antiviral-therapy/

Pan American Health Organization. (2021). Ongoing Living Update of COVID-19 Therapeutic Options: Summary of Evidence. Rapid Review, 6 May 2021. Recuperado de https://iris.paho.org/bitstream/handle/10665.2/52719/PAHOIMSEIHCOVID-1921015_eng.pdf?sequence=39\&isAllowed=y

Pandey, S., Pathak, S. K., Pandey, A., Salunke, A. A., Chawla, J., Sharma, A., Sharma, S., Thivari, P., \& Ratna, H. (2020). Ivermectin in COVID-19: What do we know?. Diabetes \& metabolic syndrome, 14(6), 1921-1922. https://doi.org/10.1016/j.dsx.2020.09.027

Portmann-Baracco, A., Bryce-Alberti, M., \& Accinelli, R. A. (2020). Antiviral and Anti-Inflammatory Properties of Ivermectin and Its Potential Use in COVID-19. Propiedades antivirales y antiinflamatorias de ivermectina y su potencial uso en COVID-19. Archivos de bronconeumologia, 56(12), 831. https://doi.org/10.1016/j.arbres.2020.06.011

Pott-Junior, H., Bastos Paoliello, M. M., Miguel, A., da Cunha, A. F., de Melo Freire, C. C., Neves, F. F., da Silva de Avó, L. R., Roscani, M. G., Dos Santos, S. S., \& Chachá, S. (2021). Use of ivermectin in the treatment of Covid-19: A pilot trial. Toxicology reports, 8, 505-510. https://doi.org/10.1016/j.toxrep.2021.03.003

Rajter, J. C., Sherman, M. S., Fatteh, N., Vogel, F., Sacks, J., \& Rajter, J. J. (2021). Use of Ivermectin Is Associated With Lower Mortality in Hospitalized Patients With Coronavirus Disease 2019: The Ivermectin in COVID Nineteen Study. Chest, 159(1), 85-92. https://doi.org/10.1016/j.chest.2020.10.009

Samaddar, A., Grover, M., \& Nag, V. L. (2020). Pathophysiology and Potential Therapeutic Candidates for COVID-19: A Poorly Understood Arena. Frontiers in pharmacology, 11, 585888. https://doi.org/10.3389/fphar.2020.585888

Schmith, V. D., Zhou, J. J., \& Lohmer, L. (2020). The Approved Dose of Ivermectin Alone is not the Ideal Dose for the Treatment of COVID-19. Clinical pharmacology and therapeutics, 108(4), 762-765. https://doi.org/10.1002/cpt.1889

Shahbaznejad, L., Davoudi, A., Eslami, G., Markowitz, J. S., Navaeifar, M. R., Hosseinzadeh, F., Movahedi, F. S., \& Rezai, M. S. (2021). Effects of Ivermectin in Patients With COVID-19: A Multicenter, Double-Blind, Randomized, Controlled Clinical Trial. Clinical. Therapeutics. https://doi.org/10.1016/j.clinthera.2021.04.007

Sharun, K., Shyamkumar, T. S., Aneesha, V. A., Dhama, K., Pawde, A. M., \& Pal, A. (2019). Current therapeutic applications and pharmacokinetic modulations of ivermectin. Veterinary world, 12(8), 1204-1211. https://doi.org/10.14202/vetworld.2019.1204-1211

Souza, M. T., Silva, M. D., \& Carvalho, R. (2010). Revisão integrativa: o que é e como fazer Integrative review: what is it? How to do it? Einstein 8(1), 102106. https://doi.org/10.1590/S1679-45082010RW1134

Trivedi, N., Verma, A., \& Kumar, D. (2020). Possible treatment and strategies for COVID-19: review and assessment. European review for medical and pharmacological sciences, 24(23), 12593-12608. https://doi.org/10.26355/eurrev_202012_24057

World Health Organization. (2021). Weekly operational update on COVID-19 31 May 2021. Recuperado de https://www.who.int/publications/m/item/weeklyoperational-update-on-covid-19-31-may-2021 
Research, Society and Development, v. 10, n. 12, e564101220835, 2021 (CC BY 4.0) | ISSN 2525-3409 | DOI: http://dx.doi.org/10.33448/rsd-v10i12.20835

Wulan, W. N., Heydet, D., Walker, E. J., Gahan, M. E., \& Ghildyal, R. (2015). Nucleocytoplasmic transport of nucleocapsid proteins of enveloped RNA viruses. Frontiers in microbiology, 6, 553. https://doi.org/10.3389/fmicb.2015.00553

Xavier, A. R., Silva, J. S., Almeida, J. P. C. L., Conceição, J. F. F., Lacerda, G. S., \& Kannan, S. (2020). COVID-19: manifestações clínicas e laboratoriais na infecção pelo novo coronavírus. J Bras Patol Med Lab, 56 (1), 1-9. https://doi.org/10.5935/1676-2444.20200049 